\title{
SISMICIDAD DE COSTA RICA DURANTE EL AÑO 2004
}

\author{
Mario Fernández A. \& Rafael Barquero P. \\ Red Sismológica Nacional (RSN: ICE-UCR) \\ Escuela Centroamericana de Geología, Apdo. 2-14, 2060 UCR \\ Fax (506) 234-2347
}

Durante el año 2004 ocurrieron 5946 temblores en Costa Rica, lo que da un promedio mensual de 495,5. Los meses de mayor actividad fueron enero, febrero, abril, mayo y noviembre; en esos meses se percibieron fuertes temblores que elevaron el número mensual de sismos de manera considerable (Cuadro 1). En abril y noviembre se generó más del $30 \%$ de la sismicidad del país en el 2004.

La generación de temblores en Costa Rica en el 2004 empezó muy alta, impulsada por la liberación de energía asociada con el terremoto de Navidad, ocurrido el 25 de diciembre del 2003 en la Zona Sur, que alteró el campo de esfuerzos de la zona y provocó gran cantidad de sismos en los meses de enero y febrero. Por ello, la actividad sísmica durante esos dos primeros meses del año se concentró en Burica, donde los habitantes vivieron momentos de gran angustia, no solo por el recuerdo de los temblores recién pasados sino también por la expectativa de un evento de igual o mayor magnitud. La frecuente ocurrencia de sismos de magnitud 4 mantenía vivos los temores de reactivación de otras fuentes sísmicas aledañas, pero afortunadamente la perturbación no pareció afectar las fallas Canoas y Longitudinal, dos muy temidas por su potencial para generar sismos grandes en la zona.
Cuadro 1

Cantidad de sismos registrados

\begin{tabular}{lcr}
\hline Mes & Cantidad & $\%$ \\
\hline Enero & 511 & 8,59 \\
Febrero & 604 & 10,16 \\
Marzo & 366 & 6,15 \\
Abril & 951 & 15,99 \\
Mayo & 538 & 9,02 \\
Junio & 299 & 5,03 \\
Julio & 367 & 6,10 \\
Agosto & 346 & 5,80 \\
Setiembre & 391 & 6,50 \\
Octubre & 333 & 5,60 \\
Noviembre & 923 & 15,50 \\
Diciembre & 317 & 5,30 \\
\hline Total & 5946 & 100 \\
\hline
\end{tabular}

En marzo la sismicidad disminuyó pero repuntó de nuevo en abril con la reanudación de la actividad sísmica en la zona de Dominical. Este hecho fue inesperado porque la sismicidad venía disminuyendo y Dominical tenía meses de estar relativamente tranquila; de hecho, allí también se había observado una paulatina disminución de la actividad. La reactivación de Dominical con un sismo de 5,1 produjo tantas 
réplicas que el total de sismos en el mes llegó a 951, el máximo pico mensual hasta el momento. Pero cuando ya casi terminaba el brote de sismicidad de Dominical, otro sobresaliente hecho ocurrió: la reactivación de la zona sísmica de Papagayo, en reposo por largos años. En su reinicio, Papagayo generó en abril un sismo de 5,8 grados y cerca de 60 réplicas.

A partir de mayo la producción sísmica empezó a disminuir y llegó a estabilizarse alrededor de los 350 temblores por mes hasta noviembre, cuando la relativa tranquilidad fue interrumpida por el fuerte sismo de Damas (Quepos) que estremeció la mayor parte del territorio nacional la madrugada del 20 de noviembre de 2004. A raíz de este evento el número de temblores llegó a 923 en noviembre, un poco menos que en abril cuando Dominical y Papagayo se reactivaron (Fig. 1).

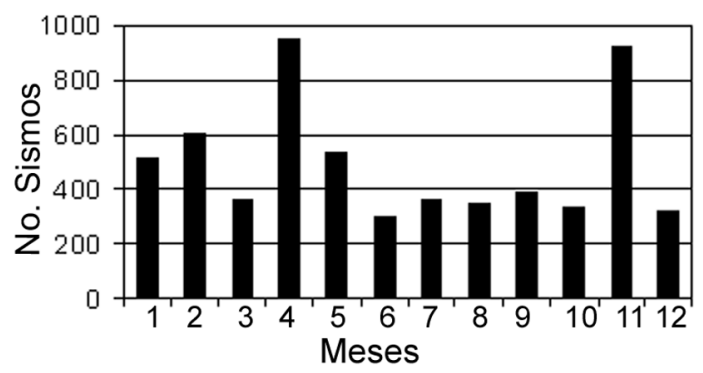

Fig. 1: Número mensual de sismos del año 2004.

\section{Profundidad de los temblores}

En cuanto a la profundidad de los temblores, 4817 fueron de foco superficial, entre los 0 y 30 km, y 1129 más profundos, lo que para Costa Rica se considera de profundidad intermedia (ver cuadro 2). A través de los meses se observó que

Cuadro 2

\begin{tabular}{lcc}
\multicolumn{3}{c}{ Profundidad de los sismos } \\
\hline Profundidad & Cantidad & $\%$ \\
\hline 0 km a $30 \mathrm{~km}$ & 4817 & 81,01 \\
Mayor a $30 \mathrm{~km}$ & 1129 & 18,99 \\
\hline Total & 5946 & 100 \\
\hline
\end{tabular}

la relación entre la cantidad de sismos superficiales y profundos se mantiene entre 70-80/30-20, lo que significa que entre el 70 y $80 \%$ de los sismos son superficiales y entre el 20 y $30 \%$ profundos. De lo anterior se concluye que los sismos provocados directamente por la colisión de las placas del Coco y Caribe (sismos de subducción) son relativamente escasos, comparados con los sismos corticales (aquellos que ocurren en la corteza de la placa Caribe y se deben a movimientos en fallas) que si son muy abundantes. También se observó que cuando aumenta o disminuye la actividad sísmica en el país, tanto los sismos superficiales como los más profundos aumentan o disminuyen en la misma proporción. Según el cuadro 2, el $81,01 \%$ fueron sismos superficiales y el18,99\% sismos intermedios y profundos.

\section{Tamaño y poder destructivo}

En el año 2004 solamente dos temblores superaron los 6 grados en la escala de magnitud (Cuadro $3)$. Por fortuna, la mayoría de los sismos más grandes y energéticos que ocurrieron en el territorio nacional se generaron en fuentes lejanas, por lo que su impacto en la población fue mínimo. Los que más impresionaron fueron los de Burica del 7 de enero y 4 de febrero y el de Damas (al Noroeste de Quepos) del 20 de noviembre, el que sin duda fue el evento más fuerte del año. Estos tres temblores provocaron destrucción y pánico. Las características más importantes de los sismos más grandes de Costa Rica del primer semestre del 2004 se muestran en el cuadro 3 .

Cuadro 3

Mayores sismos de Costa Rica en el 2004

\begin{tabular}{cccc}
\hline Fecha & Profundidad & Magnitud & Zona sísmica \\
\hline $07-01$ & 15 & 5,1 & Burica \\
$04-02$ & 12 & 5,8 & Burica \\
$07-04$ & 6,6 & 5,1 & Dominical \\
$28-04$ & 17 & 5,8 & Papagayo \\
$29-06$ & 24 & 6,1 & Papagayo \\
$20-11$ & 18 & 6,2 & Damas, \\
& & & Quepos \\
\hline
\end{tabular}


La gran mayoría de los temblores fue de magnitud inferior a los 3,5 grados $(77,3 \%$, cuadro 4). Temblores de tal magnitud rara vez son destructivos y aunque algunos llegan a ser sentidos, la mayoría de ellos solo son detectados por los instrumentos sísmicos.

Cuadro 4

Magnitud de los sismos

\begin{tabular}{lcc}
\hline 2 a 3,5 & 4599 & 77,3 \\
Mayor a 3,5 & 1347 & 22,7 \\
\hline Total & 5946 & 100 \\
\hline
\end{tabular}

\section{Las zonas más activas}

Las zonas más activas fueron: Burica, Damas (Quepos) Dominical, Quepos (sur), Pérez Zeledón, Papagayo y golfo de Nicoya (Cuadro 5, Fig. 2). Sin la menor duda, Burica fue la zona sísmica más activa en el 2004, en ella ocurrieron 505 sismos y muchos de ellos fueron eventos de magnitud superior a 4 grados (Fig. 3). Pero fue en la zona de Damas donde ocurrió la actividad más importante del año, un evento principal y muchas réplicas de las cuales hay localizadas más de 500. Dominical y Papagayo se reactivaron con fuertes temblores en abril. En el golfo y la península de Nicoya tembló pero muy intermitentemente, al igual que en otras zonas del país donde la actividad aparece en un mes pero desaparece en el siguiente. El 63,2\% de la actividad
Cuadro 5

Cantidad de sismos según zona sísmica con mayor actividad

Datos de sismos superficiales

\begin{tabular}{lcc}
\hline Zona & Cantidad & $\%$ \\
\hline Otras zonas (dispersas) & 3043 & 63,2 \\
Burica & 505 & 10,5 \\
Damas-Quepos & 500 & 10,4 \\
Dominical & 356 & 7,4 \\
Quepos (sur) & 160 & 3,3 \\
Pérez Zeledón & 100 & 2,1 \\
Papagayo & 97 & 2,0 \\
Golfo de Nicoya & 56 & 1,2 \\
\hline Total & 4817 & 100 \\
\hline
\end{tabular}

sísmica superficial ocurrió en forma muy dispersa por todo el país.

\section{Temblores percibidos}

Durante el 2004 se contabilizaron 54 temblores sentidos por la población como se ilustra en el cuadro 6 y la figura 4. En los meses de noviembre y diciembre se sintieron muchos temblores, réplicas del sismo de Quepos del 20 de noviembre, pero fue imposible saber con exactitud su número ya que muchos de ellos eran percibidos solamente cerca de la zona epicentral sin que hubiera reporte de la sacudida. Febrero y noviembre son los meses cuando se sintieron más temblores en Costa Rica porque en esos meses hubo sismos fuertes que provocaron más sismicidad en zonas del Pacifico Sur y Pacífico Central. 


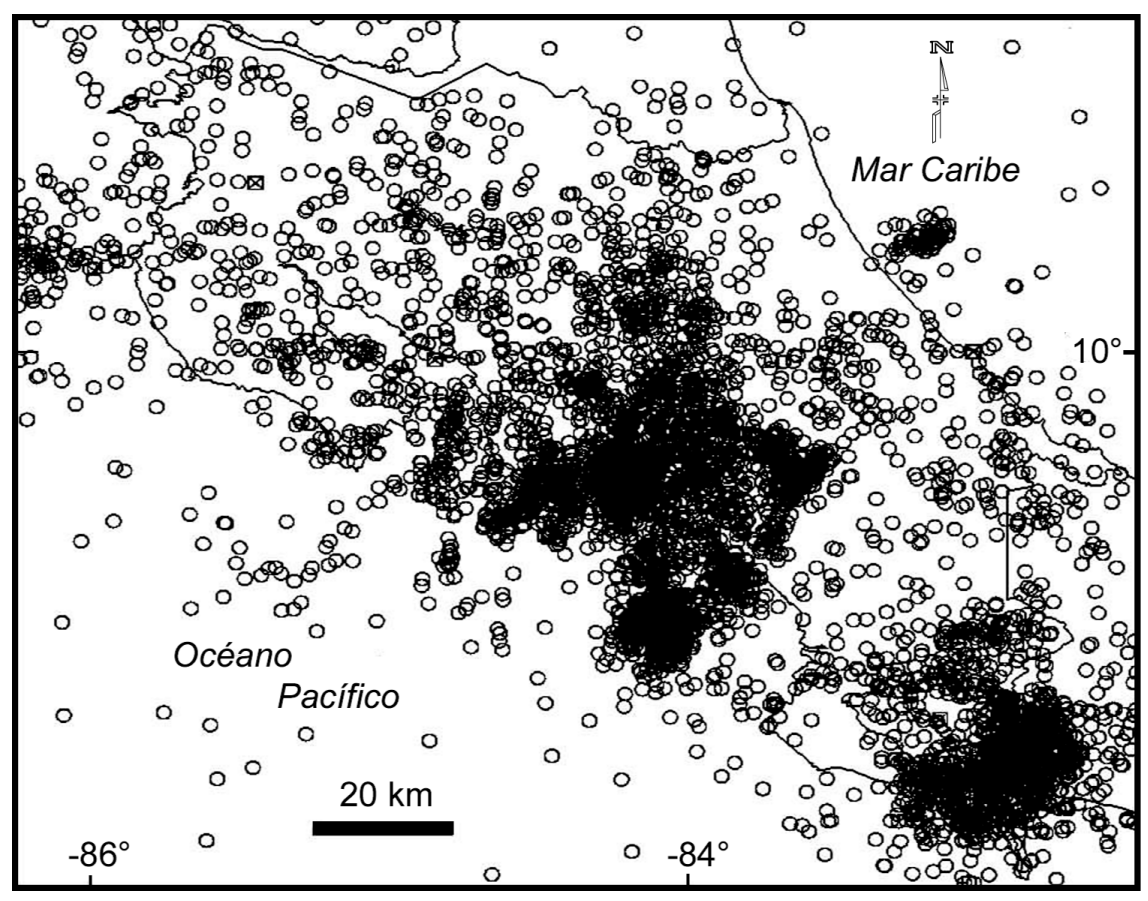

Fig. 2: Sismicidad de Costa Rica durante el año 2004.

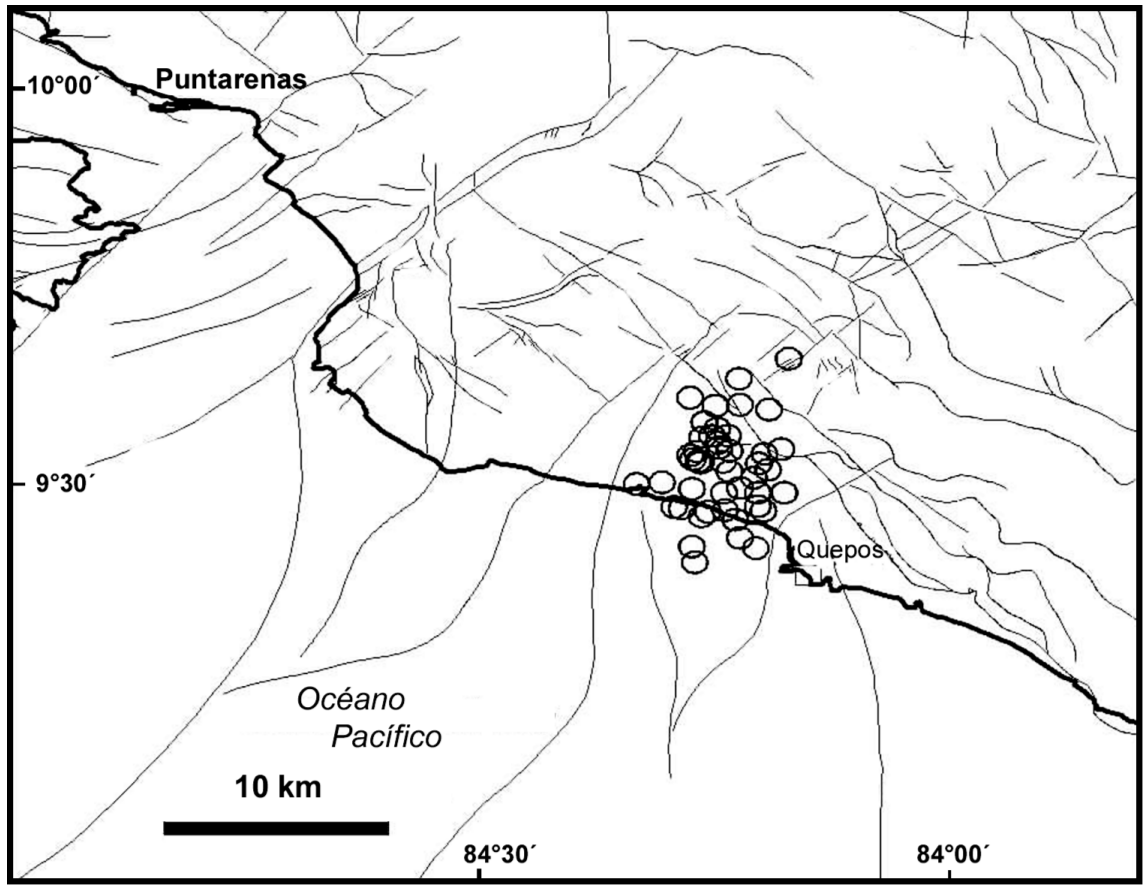

Fig. 3: Sismos en la zona de Quepos-Parrita en noviembre 2004. (Mapa base modificado de Denyer et al., 2003). 


\section{Cuadro 6}

Cantidad de sismos sentidos

\begin{tabular}{lcc}
\hline Mes & Cantidad & Estado de la sismicidad \\
\hline Enero & 4 & Normal \\
Febrero & 8 & Perturbación en Burica \\
Marzo & 4 & Brote en Escazú \\
Abril & 2 & Reactivación de Papagayo \\
Mayo & 7 & Normal \\
Junio & 5 & Normal \\
Julio & 3 & Normal \\
Agosto & 4 & Normal \\
Septiembre & 2 & Normal \\
Octubre & 2 & Normal \\
Noviembre & 13 & Normal \\
Diciembre & 3 & \\
\hline Total & 54 & \\
\hline
\end{tabular}

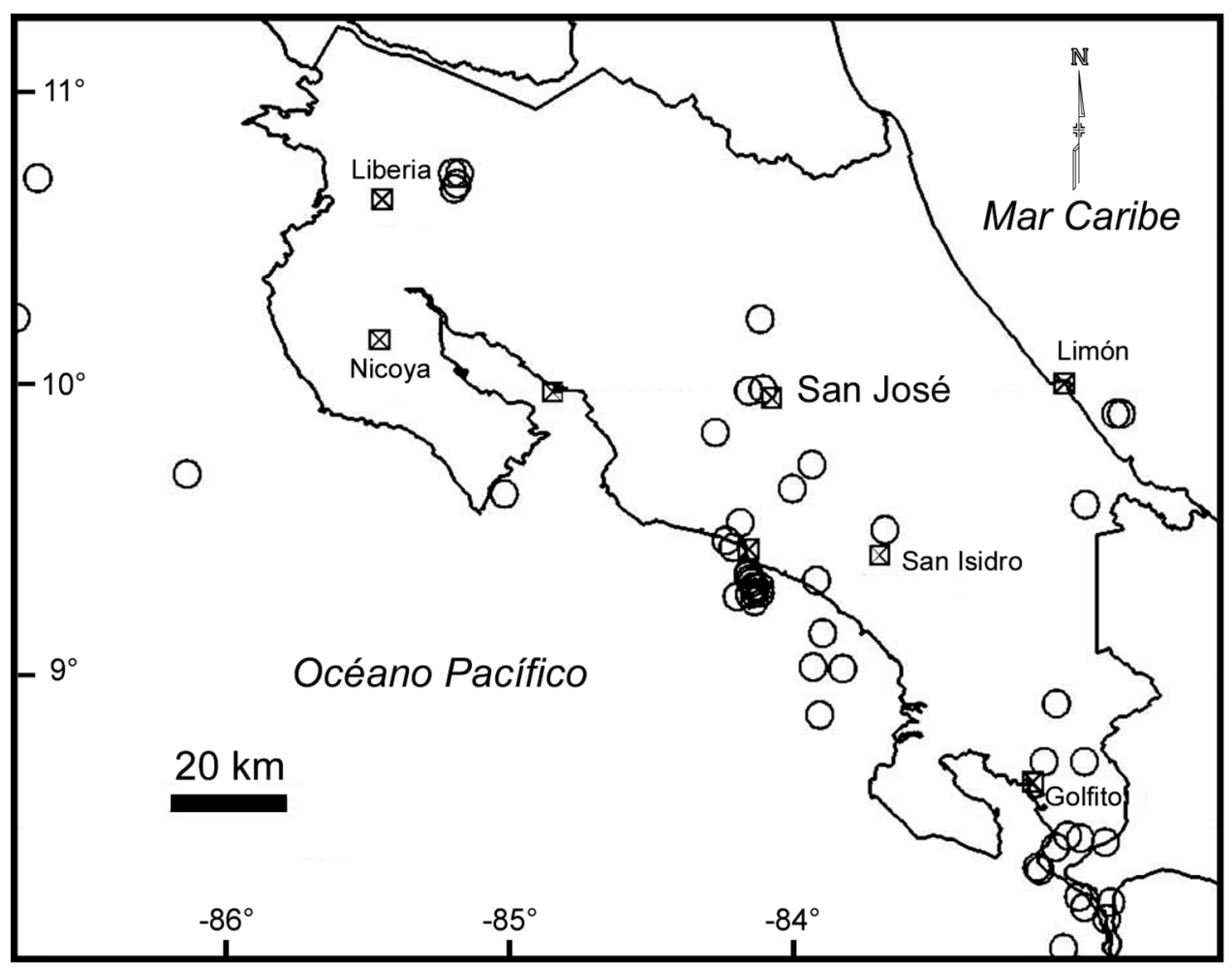

Fig. 4: Sismos reportados como sentidos durante el año 2004. 
